\title{
Effects of Proteoglycan on Dextran Sulfate Sodium-Induced Experimental Colitis in Rats
}

\author{
Sakae Ota $\cdot$ Shuichi Yoshihara $\cdot$ Keinosuke Ishido $\cdot$ Masanori Tanaka $\cdot$ \\ Keiichi Takagaki · Mutsuo Sasaki
}

Received: 20 September 2006/Accepted: 10 April 2008/Published online: 8 May 2008

(C) The Author(s) 2008

\begin{abstract}
Proteoglycans (PG) are macromolecules composed of glycosaminoglycan chains covalently attached to a protein core. In this study, we examined the effects of PG on dextran sulfate sodium (DSS)-induced experimental colitis in rats. First, to examine whether PG may ameliorate acute established DSS colitis, PG was administered orally for 5 days to the model animals. We evaluated the effects of PG on the basis of clinical symptoms, hematological analysis, macroscopic observation, and microscopic examination. We then examined whether PG administered orally to rats was detectable in their colonic lumen. After administration of PG, the colonic contents were collected, and the molecular weight of PG in the sample was analyzed by gel filtration high-performance liquid chromatography. Furthermore, we examined whether orally administered PG affected the concentrations of short-chain fatty acids (SCFAs) in the colonic feces. Orally administered PG ameliorated the clinical symptoms of bloody stools and diarrhea, and attenuated the increase in the white blood cell count in rats with established DSS colitis. Histologically, orally administered PG reduced the degree of mucosal erosion and inflammatory cell infiltration into the erosive area induced by DSS. Orally administered PG was detected
\end{abstract}

S. Ota $(\square) \cdot$ S. Yoshihara $\cdot$ K. Ishido $\cdot$ M. Sasaki

Second Department of Surgery, Hirosaki University School of Medicine, 5 Zaifu-cho, Hirosaki 036-8562, Japan

e-mail: sakaeota@cc.hirosaki-u.ac.jp

M. Tanaka

Department of Pathology, Hirosaki University School of Medicine, 5 Zaifu-cho, Hirosaki 036-8562, Japan

K. Takagaki

Department of Biochemistry, Hirosaki University School

of Medicine, 5 Zaifu-cho, Hirosaki 036-8562, Japan in rat colon, although its molecular weight was slightly decreased. Orally administered PG significantly increased the concentration of total SCFAs and $n$-butyrate in rat colonic feces. This is the first study to indicate that exogenous PG ameliorates experimental colitis, suggesting the potential usefulness of PG for clinical treatment of colitis.

Keywords Xogenous proteoglycan .

Experimental colitis · Drug therapy · Ulcerative colitis

$\begin{array}{ll}\text { Abbreviations } \\ \text { CS } & \text { Chondroitin sulfate } \\ \text { DSS } & \text { Dextran sulfate sodium } \\ \text { GAG } & \text { Glycosaminoglycan } \\ \text { HPLC } & \text { High-performance liquid chromatography } \\ \text { PG } & \text { Proteoglycans } \\ \text { SCFAs } & \text { Short-chain fatty acids } \\ \text { UC } & \text { Ulcerative colitis }\end{array}$

\section{Introduction}

Proteoglycans (PG) are macromolecules with molecular weights of several million composed of glycosaminoglycan (GAG) chains attached covalently to a protein core $[1,2]$. PG may be present intracellularly at the cell surface or in the extracellular matrix. The biological roles of PG are highly diverse, ranging from relatively simple mechanical support to more intricate, as yet rather poorly understood effects on various cellular processes, such as adhesion, motility, and proliferation, as well as differentiation and tissue morphogenesis [1,2].

Chondroitin sulfate (CS) is a major GAG component of PG [1, 2]. Inflammatory conditions, such as necrotizing 
enterocolitis and inflammatory bowel diseases, result in a loss of CS in the mucosa [3, 4]. It is also postulated that CS partially inhibits leukocyte elastase activity and inhibits connective tissue mast cells $[5,6]$. CS is used as a safe drug in patients with osteoarthritis [7]. In humans, orally administered CS is believed to be degraded in the colon [8, 9]. Generally, dietary carbohydrates that escape digestion in the small intestine undergo bacterial fermentation in the colon and generate short-chain fatty acids (SCFAs). One of the SCFAs, $n$-butyrate, is used in the treatment of distal ulcerative colitis (UC) $[10,11]$.

As described above, the functions of endogenous PG or $\mathrm{CS}$, and the digestion of exogenous CS, have been studied. However, details of the function and digestion of exogenous $\mathrm{PG}$ in vivo remain unknown. Therefore, we investigated whether PG may ameliorate the acute colitis induced by dextran sulfate sodium (DSS) in rats [12].

\section{Materials and Methods}

\section{Animals and Housing Conditions}

Male Wistar rats weighing 270-300 g were purchased from CLEA Japan (CLEA Japan, Inc., Tokyo, Japan). These animals were housed in wire mesh-bottom cages in a room with controlled temperature $\left(22 \pm 2^{\circ} \mathrm{C}\right)$, humidity $(60 \%)$, and a light-dark cycle (12 h:12 h). Standard rodent chow pellets (Oriental MF; Oriental Yeast Co., Ltd., Tokyo, Japan) and tap water were provided ad libitum. All animal experiments in this study followed the Guidelines for Animal Experimentation of Hirosaki University.

\section{Preparation of PG and CS from Salmon Nasal Cartilage}

PG from salmon nasal cartilage was prepared by the method described by Majima et al. [13]. CS was prepared by digestion of PG with Actinase E (Kaken Pharmaceutical Co., Tokyo, Japan) and endo- $\beta$-xylosidase [14]. Briefly, PG was treated with actinase $\mathrm{E}$ in $0.1 \mathrm{M}$ Tris- $\mathrm{HCl}$ buffer $(\mathrm{pH}$ 8.0) containing $10 \mathrm{mM} \mathrm{CaCl} 2$ at $50^{\circ} \mathrm{C}$ for $12 \mathrm{~h}$. The precipitate obtained with $4 \mathrm{vol}$ of $\mathrm{NaCl}$-saturated ethanol was digested with endo- $\beta$-xylosidase in $0.1 \mathrm{M}$ sodium acetate

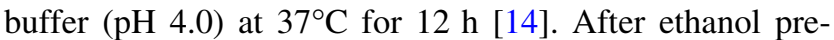
cipitation, CS bearing a linkage region at its reducing terminal sites was obtained. The monosaccharide and protein composition of $\mathrm{PG}$, expressed as a molar ratio against hexosamine at 1.00 , was uronic acid 0.99 , sulfate 0.67 , and protein $6.99 \%(\mathrm{w} / \mathrm{w})$. Analysis of unsaturated disaccharides of this PG yielded $\Delta \mathrm{Di}-0 \mathrm{~S} 15.1 \%, \Delta \mathrm{Di}-6 \mathrm{~S}$ $59.4 \%, \Delta \mathrm{Di}-4 \mathrm{~S} 25.1 \%, \Delta \mathrm{Di}-\mathrm{diSD} 0.3 \%$, and $\Delta \mathrm{Di}$-triS $0.1 \%$ [13]. PG and CS were dissolved in distilled water (DW) to produce a $1 \%$ solution.

\section{Effect of PG on Established DSS Colitis}

A total of 62 rats was included in this experiment. Colitis was induced by oral DSS, as described by Okayasu et al. [12] with modification. The normal group $(n=10)$ was given DW for 10 days. The control, CS, and PG groups were given $4 \%$ DSS (mean molecular weight 5,000, sulfur content $18.5 \%$, degree of sulfation 2.4; Wako Pure Chemical Industries, Ltd., Osaka, Japan) solution for the first 5 days to induce colitis and 1\% DSS solution for the next 5 days to maintain the colitis. The PG and CS groups ( $n=16$ each) received $1 \%$ PG solution $1.5 \mathrm{ml}$ or $1 \% \mathrm{CS}$ solution $1.5 \mathrm{ml}$ orally twice a day for the last 5 days. The control group ( $n=20$ ) was given DW $1.5 \mathrm{ml}$ orally twice a day for the last 5 days. The quantity of DSS ingested and the stool conditions were monitored daily. Stool consistency and blood content were scored from observations made in a pilot study by the method of Cooper et al. [15] and subsequently modified: grade 0 , normal stools with well-formed pellets; grade 1, loose stools of pasty and semi-formed appearance; grade 2, diarrhea with liquid stools that stick to the anus; grade 3, stools with blood; grade 4 , gross bleeding with constant bleeding from the anus.

On days 1,5 , and 10, blood was withdrawn from the tail vein of rats under ether anesthesia with a 1-ml syringe and a 26-gauge needle. The blood was collected into a vial containing EDTA (Sysmex Co., Ltd., Tokyo, Japan). For hematological examination, the erythrocyte count (RBC) and leukocyte count (WBC) were determined with a blood cell counter (Sysmex F-520; Sysmex Co.).

On day 10 , rats were killed by an intraperitoneal overdose of Nembutal (Dainippon Pharmaceutical Co., Ltd., Osaka, Japan). After sacrifice, the colon from the colocecal junction to the anal verge was excised. The colon was opened longitudinally from the mesenteric side, dilated, and fixed in neutral buffered formalin. The colon was cut longitudinally in the midline for $5 \mathrm{~cm}$ from the anal verge and used for microscopic assessment. Specimens were embedded in paraffin. Sections $4-\mu \mathrm{m}$ thick were stained with hematoxylin and eosin. All sections were examined blind by an experienced gastrointestinal pathologist (M.T.), who determined the ratio of the erosive area to total mucosa and the infiltration degree of neutrophils, mononuclear cells, and eosinophils in the erosive area. The degree was classified as minimal $(=0)$, mild $(=1)$, moderate $(=2)$, or severe $(=3)$, and the data for the lamina propria mucosae and submucosa were recorded separately.

\section{Detection of PG in Colonic Lumen}

A total of nine rats was included in this experiment. Rats were deprived of chow pellets for $24 \mathrm{~h}$. One percent PG 
solution $1.5 \mathrm{ml}$ or $1 \% \mathrm{CS}$ solution $1.5 \mathrm{ml}$ was administered orally to rats in the test groups. Control rats received $1.5 \mathrm{ml}$ of DW. At 1, 2, and 3 after administration, rats were killed by an intraperitoneal overdose of Nembutal.

After sacrifice, the colon from the colo-cecal junction to the anus was excised. Both ends of the colon were ligated with forceps, and the serosal side of the colon was washed with sterile saline. The colonic contents were rinsed out in $10 \mathrm{ml}$ of DW containing protease inhibitors (1 mM PMSF, $5 \mathrm{mM}$ benzamidine, $1 \mathrm{mM}$ EDTA, $10 \mathrm{mM} N$-ethylmaleimide) and suspended. The suspension was centrifuged at $15,000 \mathrm{~g}$ for $15 \mathrm{~min}$ at $4{ }^{\circ} \mathrm{C}$. The collected supernatants were applied to a reverse-phase column (Sep-Pak Plus C18; Waters Co., Ireland) and the extract liquid $(1.0 \mathrm{ml})$ was lyophilized. This concentrated sample thus lyophilized was dissolved in $0.2 \mathrm{M} \mathrm{NaCl}$ buffer $(100 \mu \mathrm{l})$ and subjected to gel filtration by high-performance liquid chromatography (HPLC) (model L-6200; Hitachi Ltd., Tokyo, Japan).

Size-fractionation HPLC was performed on a Shodex OHpak SB-805 column $\left(0.8 \times 30 \mathrm{~cm}^{2}\right.$; Showa Denko Co., Tokyo, Japan) with $0.2 \mathrm{M} \mathrm{NaCl}$ at a flow rate of $0.5 \mathrm{ml} / \mathrm{min}$. The column temperature was $30^{\circ} \mathrm{C}$. Fractions were assayed for uronic acid by the carbazole reaction [16].

\section{Effect of PG on SCFAs in Colonic Feces}

A total of 18 rats were included in this experiment. Standard rodent chow pellets and tap water were provided ad libitum. The PG group $(n=6)$ and the CS group $(n=6)$ received $1 \% \mathrm{PG}$ solution $1.5 \mathrm{ml}$ or $1 \% \mathrm{CS}$ solution $1.5 \mathrm{ml}$ twice a day for 5 days. The control group $(n=6)$ received $1.5 \mathrm{ml} \mathrm{DW}$ twice a day for 5 days. On day $6,12 \mathrm{~h}$ after the last administration, rats were killed, and the colon from the colo-cecal junction to the anus was excised. The contents of the colon were collected and weighed in a test tube. The contents were suspended in 3 vol of DW and centrifuged at $3,000 \mathrm{~g}$ for $15 \mathrm{~min}$. The supernatants $(100 \mu \mathrm{l})$ were used for analysis of SCFA by HPLC.

Analysis of SCFA was performed by the method described previously [17, 18]. Briefly, standard SCFAs (GL Science Inc., Tokyo, Japan) and SCFAs in the samples, including ethyl butyrate as an internal standard, were labeled with an SCFA labeling kit (YMC Co., Tokyo, Japan), separated on a reverse-phase column (YMC-Pak FA, $0.6 \times 25 \mathrm{~cm}^{2}$; YMC Co.) and detected by UV fluorescence at $400 \mathrm{~nm}$.

\section{Statistical Analysis}

All results are presented as means \pm SEM. Overall statistical difference was determined by one-way analysis of variance, and post-test comparisons between the two groups were performed with the Student-Neuman-Keuls test.

\section{Results}

Effect of PG on Symptom Score and Hematological Values

During this experiment, there was no significant difference between the groups in the quantity of $4 \%$ DSS or $1 \%$ DSS consumed (data not shown). First, we observed colitis symptoms daily and scored them (Fig. 1). The normal group did not show colitis symptoms during this experiment (average score $0 \pm 0$ ). DSS caused severe diarrhea or gross bleeding from the anus in the DSS ingestion groups. On day 5, when treatment was started, the scores in the DSS ingestion groups did not differ significantly. The score in the control group increased daily, but from day 7 it showed a mild improvement. On days 9 and 10, the average scores in the control group were $2.65 \pm 0.19$ and $2.50 \pm 0.26$, respectively. The score in the PG group increased daily, but from day 6 the symptoms of colitis improved markedly. On days 9 and 10, the symptoms in the PG group were significantly improved, with average scores of $1.75 \pm 0.19$ and $1.56 \pm 0.30$, respectively $(P<0.05$ vs. control). On days 9 and 10, the symptoms in the CS group were not significantly improved, with average scores of $2.37 \pm 0.23$ and $2.06 \pm 0.19$, respectively.

We then examined blood from the tail vein (Table 1). On day 1 , there were no differences in $\mathrm{RBC}$ and $\mathrm{WBC}$ between the groups. On day 5, RBC and WBC in the DSStreated groups were not significantly different. On day 10 , $\mathrm{RBC}$ in the PG group was significantly higher than in the control group, and WBC in the PG group was significantly lower than in the control group. However, RBC and WBC in the CS group on day 10 showed no difference from the control group.

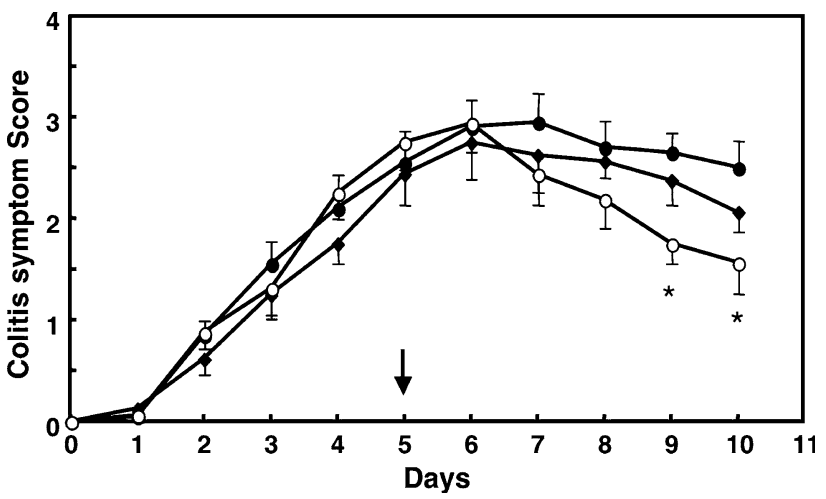

Fig. 1 Effect of PG on colitis symptom score. Rats were given $4 \%$ DSS solution for the first 5 days, followed by $1 \%$ DSS solution for the last 5 days, and colitis symptoms induced by DSS were monitored daily according to the score described in "Materials and methods." The arrow indicates the day that treatment with PG or CS was started. $\boldsymbol{\bullet}$, Control group; $\bullet$, CS group; $\bigcirc$, PG group. ${ }^{*} P<0.05$ vs. control 
Table 1 The time course of WBCs and RBCs count in the tail vein

\begin{tabular}{cccl}
\hline & Day 1 & Day 5 & Day 10 \\
\hline WBC $\times 10^{3} / \mathrm{mm}^{3}$ (leucocyte cell count) \\
Normal & $124.2 \pm 4.8$ & $123.3 \pm 6.2$ & $127.1 \pm 5.4$ \\
Control & $132.3 \pm 7.7$ & $255.2 \pm 14.9$ & $248.6 \pm 13.3$ \\
CS & $121.9 \pm 5.7$ & $257.9 \pm 8.6$ & $230.7 \pm 10.3$ \\
PG & $134.1 \pm 10.8$ & $251.5 \pm 15.8$ & $201.6 \pm 14.0^{*}$ \\
RBC $\times 10^{4} / \mathrm{mm}^{3}$ (erythrocyte cells count) & \\
Normal & $773.2 \pm 11.0$ & $782.3 \pm 10.8$ & $775.7 \pm 9.2$ \\
Control & $756.0 \pm 6.9$ & $695.7 \pm 14.1$ & $564.6 \pm 32.2$ \\
CS & $750.3 \pm 10.6$ & $698.8 \pm 15.4$ & $588.4 \pm 28.3$ \\
PG & $745.5 \pm 11.4$ & $685.8 \pm 17.2$ & $673.4 \pm 28.9^{*}$ \\
\hline
\end{tabular}

Each value represents the mean \pm SEM

$* P<0.05$ vs. control

Effect of PG on Macroscopic and Histopathologic Changes

First, the severity of DSS-induced colitis was compared macroscopically. As shown in Fig. 2a, DSS caused shortening of the colon and macroscopic ulceration in the distal colon. These changes were milder in the PG and CS groups. DSS caused significant shortening of the colon in the control group $(15.31 \pm 0.29 \mathrm{~cm})$ compared with the normal group $(18.90 \pm 0.38 \mathrm{~cm})$. However, oral administration of PG significantly ameliorated the shortening of colon length $(17.03 \pm 0.32 \mathrm{~cm})$ compared with the control group. Oral administration of CS $(15.98 \pm 0.29 \mathrm{~cm}) \mathrm{did}$ not significantly ameliorate the shortening in comparison with the control group (Fig. 2b).

Next, the severity of mucosal damage and mucosal inflammation was evaluated by histopathologic observation (Figs. 3-5). For histopathologic evaluation, we used a specimen of the distal colon taken $5 \mathrm{~cm}$ from the anal verge because, as shown in Fig. 2a, macroscopic mucosal damage was limited to the distal $5 \mathrm{~cm}$ of the colon. The total length of mucosa in this section did not differ significantly among the groups (normal group, $49.20 \pm 0.74 \mathrm{~mm}$; control group, $47.89 \pm 0.85 \mathrm{~mm}$; CS group, $48.66 \pm 0.50 \mathrm{~mm}$; PG group, $47.26 \pm 0.57 \mathrm{~mm})$. In the normal group, no mucosal damage or erosion was observed. However, areas of erosion were observed in the DSS-treated group, as shown in Fig. 3a. The erosion area as a proportion of the total mucosa was $36.31 \pm 3.13 \%$ in the control group. Oral administration of PG significantly reduced the area of erosion compared with the control group. However, oral CS did not significantly reduce the area of erosion (Fig. 3b).

We also evaluated infiltration of inflammatory cells into the area of erosion. Neutrophils, eosinophils, and mononuclear cells were observed in the erosive area and were localized in the lamina propria mucosae and submucosa. In the lamina propria mucosae, oral administration of PG significantly reduced the infiltration of neutrophils and increased the infiltration of eosinophils compared with the control group (Figs. 4 and 5a). In the submucosa, oral administration of PG significantly reduced the infiltration of neutrophils compared with the control group (Fig. 5b).
Fig. 2 Effect of PG on macroscopic changes. For details of experimental design, see "Materials and methods." (a) Gross appearance of the luminal side of the distal colon. The bar indicates $5 \mathrm{~cm}$ length. (b) The length of the colon from the anal verge to the colo-cecal junction was measured $(* P<0.05$ vs. control)
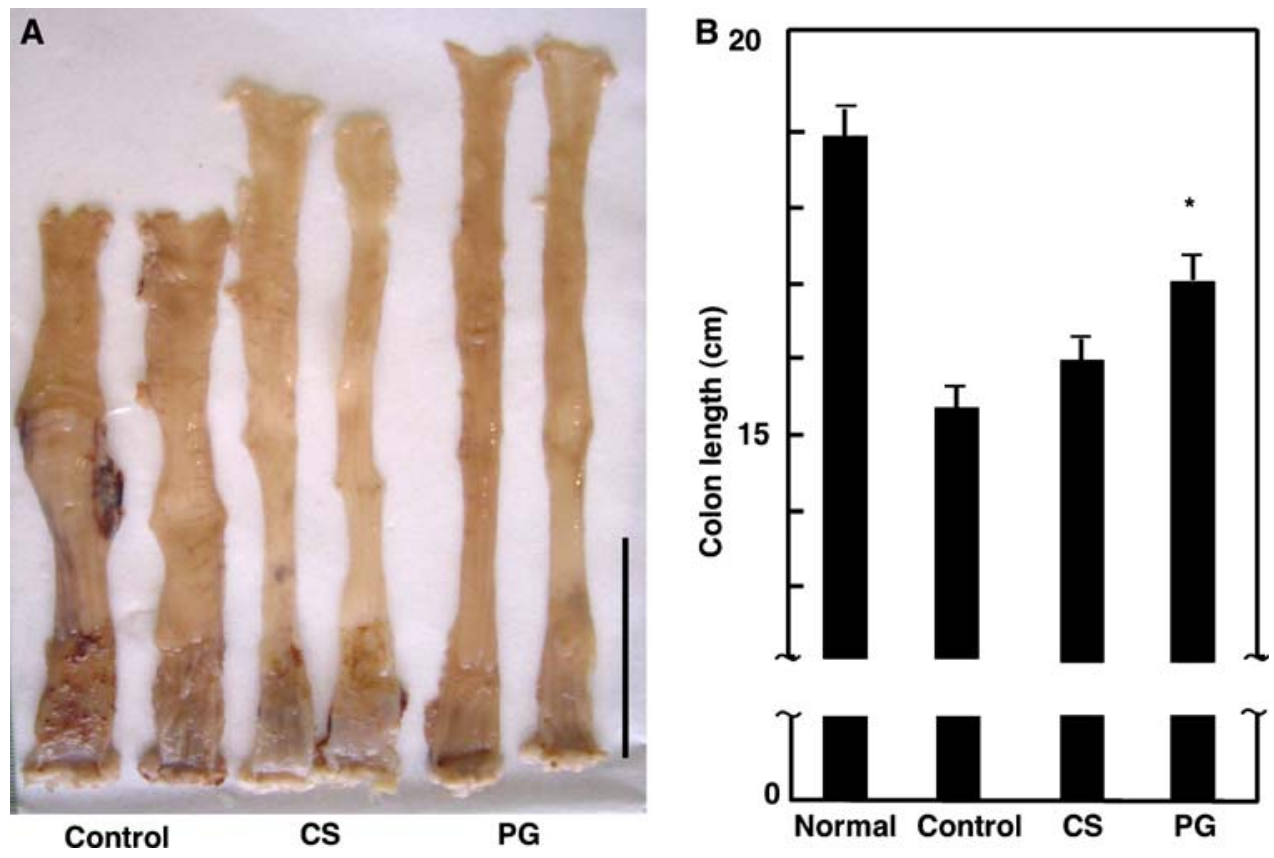

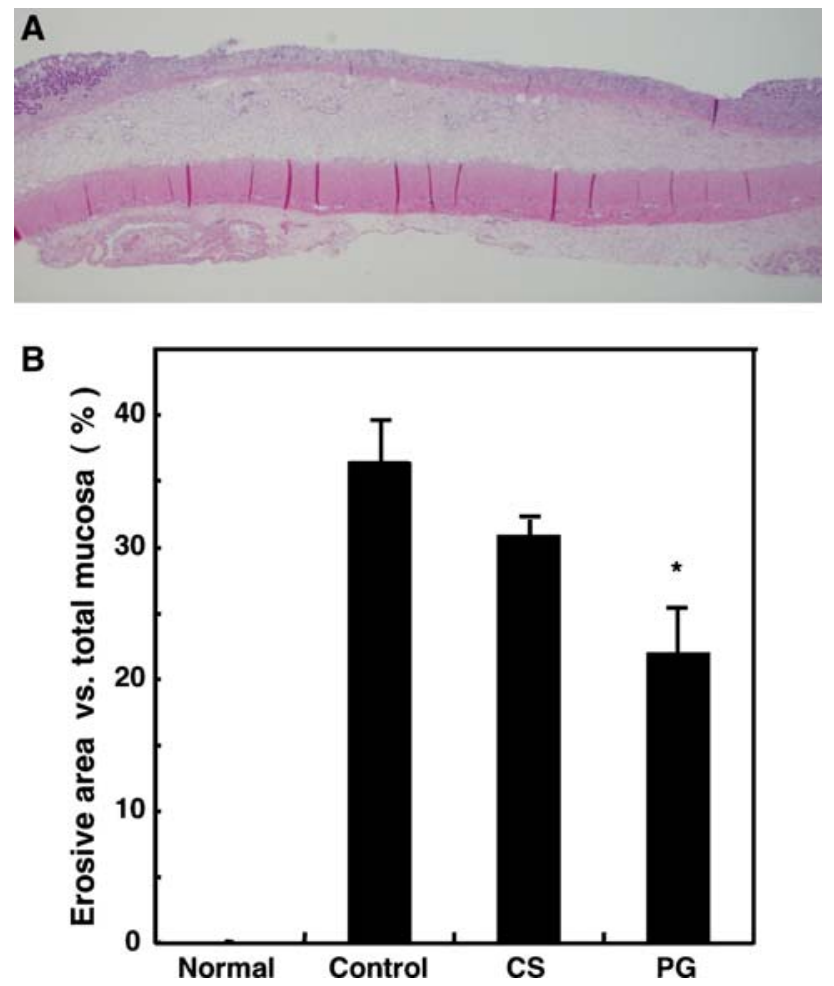

Fig. 3 Effect of PG on erosive area induced by DSS. For details of experimental design, see "Materials and methods." (a) Microphotograph of the erosive area in the control group (H\&E staining; original magnification, $4 \times$ ). (b) The percentage of the erosion area relative to the total mucosa was calculated ( ${ }^{*} P<0.05$ vs. control)

\section{Detection of PG in the Colonic Lumen}

We examined whether orally administered PG and CS was detectable in the rat colon. Uronic acid-containing materials were not detectable in samples from the colonic contents of control rats (Fig. 6a) or at 1 and $2 \mathrm{~h}$ after oral administration of PG or CS (data not shown). At $3 \mathrm{~h}$ after oral administration of $\mathrm{PG}$, high-molecular-weight uronic acid-containing materials were detected in the colonic content sample near the void volume area; however, a lowmolecular-weight peak was also detected (Fig. 6c). On the other hand, at $3 \mathrm{~h}$ after oral administration of CS, only the low-molecular-weight peak was detectable (Fig. 6b).

\section{Effect of PG on SCFAs in Colon Feces}

We examined whether orally administered PG or CS can affect the concentration of SCFAs in colon feces. The concentrations of individual SCFAs in colon feces are shown in Table 2 . The concentration of $n$-butyrate was significantly increased by oral administration of PG $(P<0.05$ vs. control). The concentration of acetate was significantly increased by oral administration of CS $(P<0.05$ vs. control). The total concentration of SCFAs

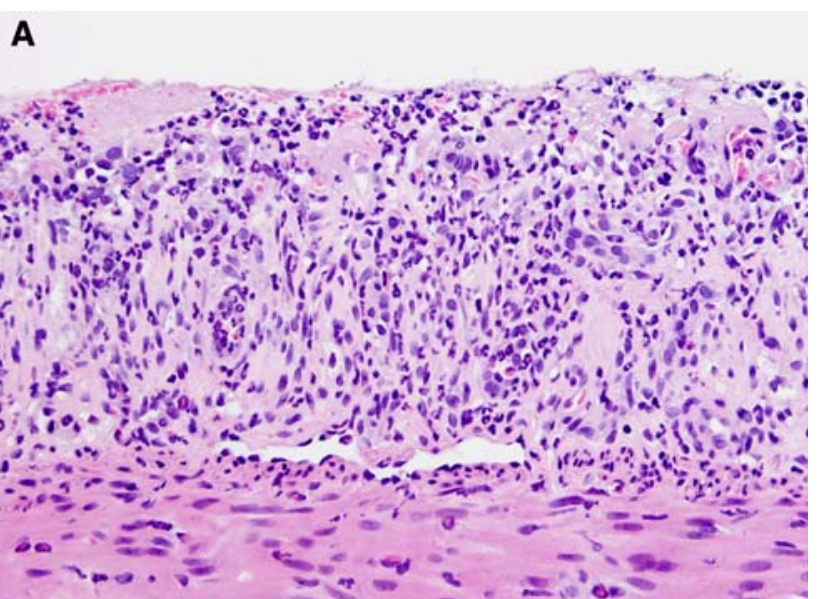

B

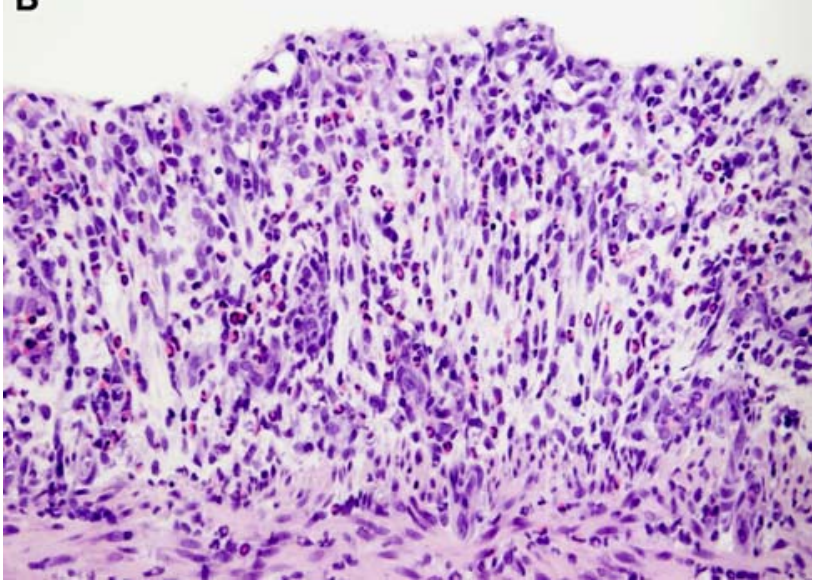

Fig. 4 Inflammatory cell infiltration into the erosive area. For details of experimental design, see "Materials and methods." Representative microphotographs of lamina propria mucosa in the erosive area of the control group (a) and that of the PG group (b) (H\&E staining; original magnification, $40 \times$ ). In the control group (a), many neutrophils had infiltrated, whereas eosinophil infiltration was remarkable in the PG group (b)

was significantly increased by oral administration of PG $(P<0.05$ vs. control), but was not significantly affected by oral administration of CS.

\section{Discussion}

In this study using rats with DSS-induced colitis, oral administration of PG ameliorated the clinical symptoms of bloody stools and diarrhea, and attenuated the increase in the WBC count. Histologically, orally administered PG reduced the degree of mucosal erosion and inflammatory cell infiltration into the erosive area induced by DSS. Orally administered PG was more resistant than CS to degradation in vivo. Orally administered PG significantly increased the concentrations of $n$-butyrate and total SCFAs in colon feces. We suggest that the anti-inflammatory effect of PG on DSS- 

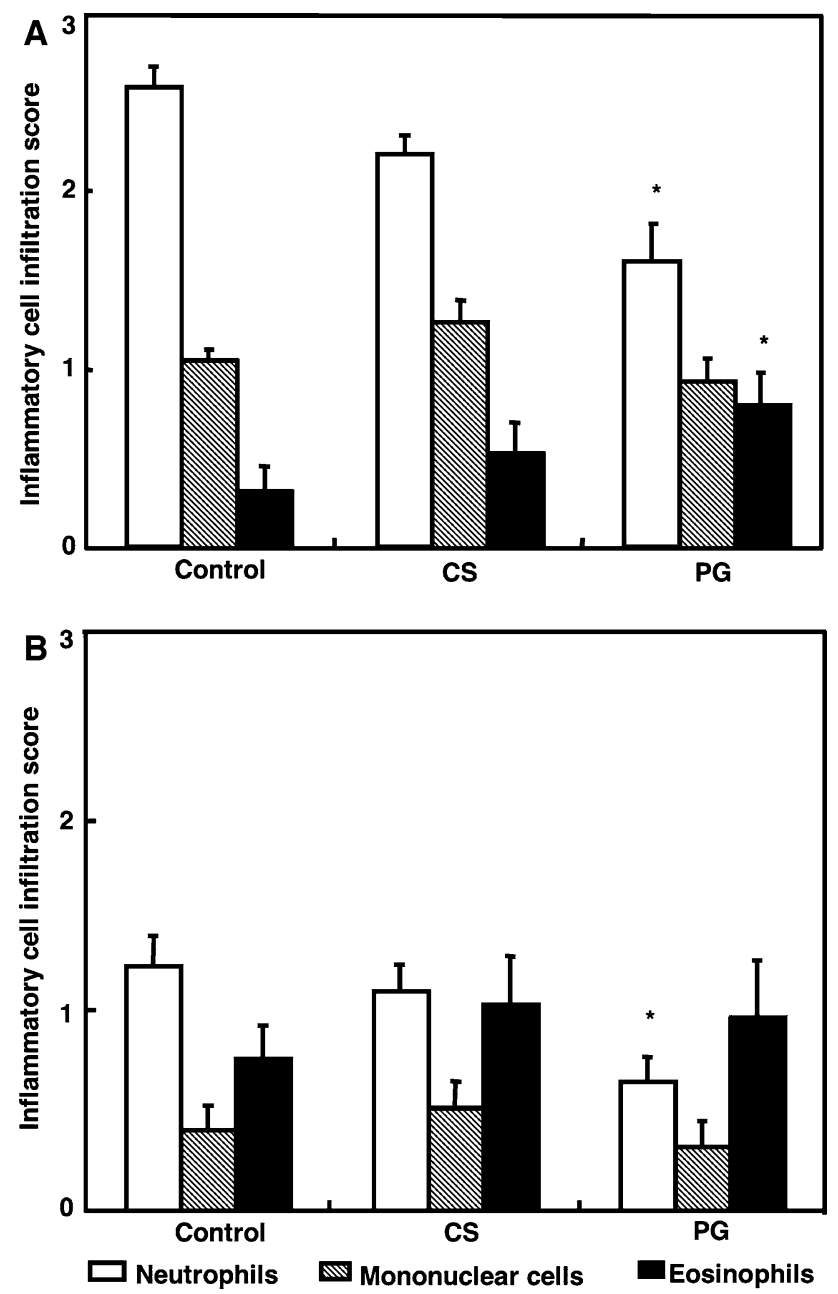

Fig. 5 Effect of PG on inflammatory cell infiltration into the erosive area. The inflammatory cell infiltration was scored in a blind manner, as described in "Materials and methods." Mean values for inflammatory cell infiltration into the lamina propria mucosa (a) and inflammatory cell infiltration into the submucosa (b) are plotted $(* P<0.05$ vs. control $)$

induced colitis may be due to the increase of $n$-butyrate and that for this role the structure of PG is important.

DSS-induced experimental colitis resembles human UC [12], is reproducible, and seems to be an adequate model for verifying the efficacy of drugs in preventing or healing colitis lesions [19, 20]. Overgrowth of Bacteroides and Clostridium species has been demonstrated [12], and a topical toxic effect of DSS on colonic epithelial cells has also been proposed [21]. However, the exact mechanism of DSS-induced mucosal injury is not fully understood. Consequently, we designed a rat colitis model involving administration of $4 \%$ DSS solution to induce the colitis and $1 \%$ DSS solution to maintain the colitis.

The earliest lesions in acute DSS colitis appear to be progressive loss and dropout of crypts, starting with loss of the basal one-third of the crypt and progressing to formation
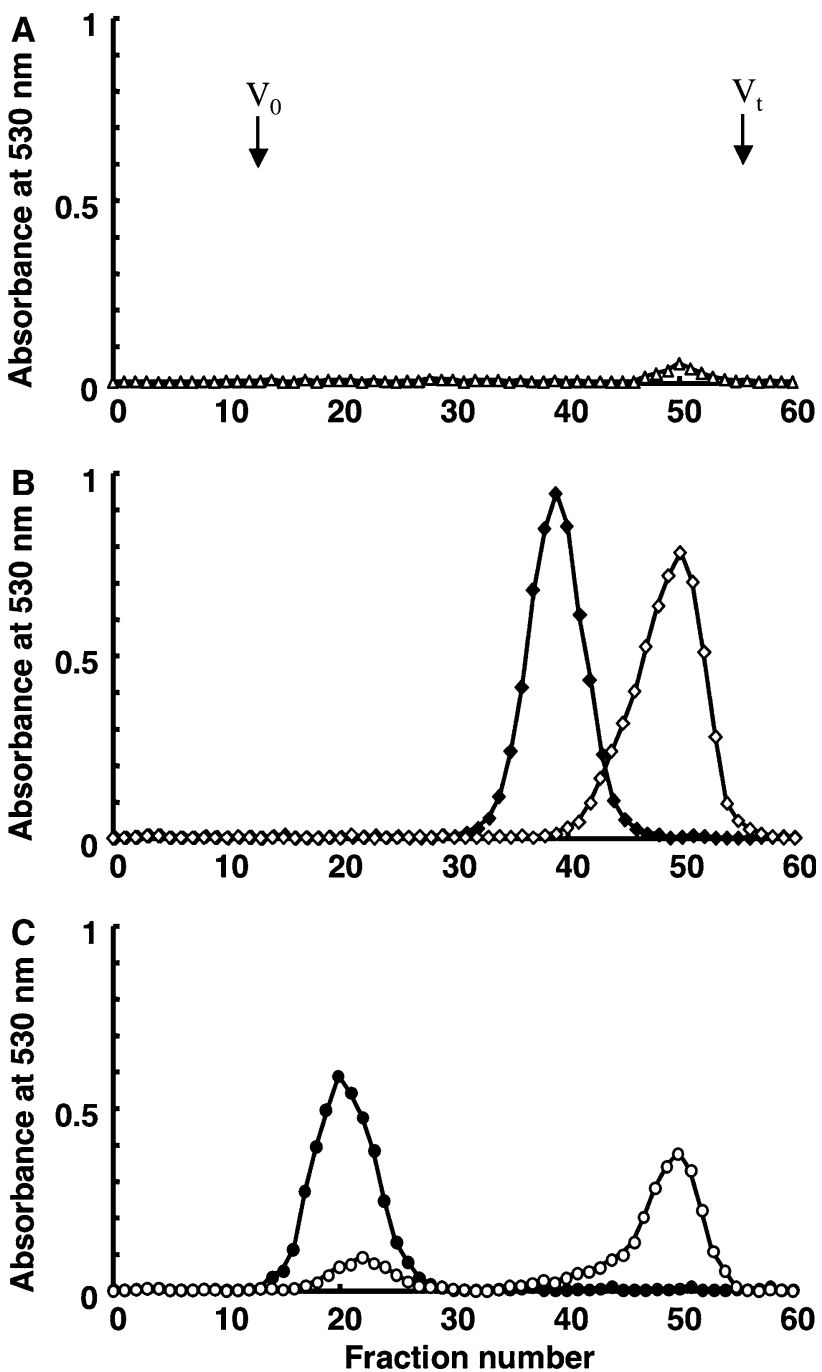

Fig. 6 Detection of orally administered PG in the colonic content of rats. Colonic content was subjected to gel-filtration HPLC on a Shodex OHpak SB-805 column, as described in "Materials and methods." (a) $\triangle$, control. (b) $\diamond$, CS, $\diamond$, administered CS. (c) $\bullet$, PG, $\bigcirc$, administered PG. $V_{0}$, void volume. $V_{\mathrm{t}}$, total volume. Uronic acidcontaining materials were not detected in samples from the colonic contents of control rats (a). After oral administration of PG, highmolecular-weight uronic acid-containing materials were detected in the colonic content sample near the void volume area; however, a low-molecular-weight peak was also detected (c). On the other hand, after oral administration of CS, only the low-molecular-weight peak was detected (b)

of erosions [15]. Therefore, we examined inflammatory cell infiltration in the most severe lesions (erosive area). In acute DSS colitis, the inflammatory infiltrate in the erosive area consists of neutrophils, lymphocytes, and plasma cells $[12,15,19]$. Oral administration of PG significantly reduced neutrophil infiltration into the lamina propria mucosae and submucosa in the areas of erosion induced by DSS (Fig. 4c, d), suggesting that PG may have an anti-inflammatory effect.

Oral administration of PG significantly increased eosinophil infiltration into the lamina propria mucosae in the areas 
Table 2 The concentration of SCFAs in the colon feces

\begin{tabular}{lccc}
\hline & \multicolumn{1}{l}{ Control } & \multicolumn{1}{l}{ CS } & \multicolumn{1}{l}{ PG } \\
\hline Lactate & $1.56 \pm 0.84$ & $1.38 \pm 0.97$ & $1.48 \pm 0.39$ \\
Acetic acid & $9.82 \pm 1.90$ & $17.56 \pm 1.66^{*}$ & $13.05 \pm 1.14$ \\
Propionic acid & $6.07 \pm 1.02$ & $7.13 \pm 1.55$ & $9.30 \pm 0.93$ \\
$n$-butyrate & $9.77 \pm 2.02$ & $11.47 \pm 2.00$ & $18.22 \pm 1.96^{*}$ \\
Total SCFAs & $25.68 \pm 4.09$ & $36.18 \pm 3.18$ & $39.89 \pm 3.89^{*}$
\end{tabular}

Each value represents the mean \pm SEM ( $\mu \mathrm{mol} / \mathrm{g}$ feces)

$* P<0.05$ vs. control

The total SCFAs concentration indicates the sum of the acetic acid, propionic acid, and $n$-butyrate concentrations

of erosion (Figs. 4b and 5a). In human UC, although the significance of eosinophil infiltration is unclear, the infiltration is significantly less marked in patients requiring surgery than in those responding to medical treatment [22]. In DSS colitis, the degree of eosinophil infiltration does not reflect the severity of the disease or the extent of tissue damage [23]. Eosinophils may act as homeostastic immune modulators and play an anti-inflammatory role, releasing aryl sulfatase $\mathrm{B}$, phospholipase $\mathrm{D}$, histaminase, and other chemical mediators [24-27]. The eosinophils in the human colonic mucosa account for more overall phagocytic activity than either neutrophils or macrophages [28, 29]. If neutrophil infiltration is reduced in erosive areas, eosinophils may play a major role in defense against invasion of enteric bacteria into the ulcerated colonic mucosa.

In this experiment, we evaluated the leukocyte and erythrocyte count in venous blood from the tail vein. Oral administration of PG attenuated the increase in the leukocyte count (Table 1), and this result was in concern with the mucosal and submucosal neutrophil infiltration. PG ameliorated the symptoms of colitis (Fig. 1) and attenuated the decrease in erythrocyte count caused by bleeding (Table 1). This bleeding is likely to arise from erosion formation, and our results showed that PG reduced the area of erosion (Fig. 3b).

Oral administration of CS did not produce the effects described above to any significant extent. This difference may have been due to the concentration of SCFAs in colonic feces. Orally administered PG increased the concentration of $n$-butyrate, whereas CS increased the concentration of acetate (Table 2). Acetate and propionate are absorbed and transported to the liver for gluconeogenesis (propionate) or to various tissues as fuel (acetate), whereas butyrate is oxidized by the colonic epithelium [30]. There is evidence that SCFAs in colon feces are decreased in human UC and also in DSS-induced colitis [31, 32]. Use of $n$-butyrate enema has been proposed for the treatment of refractory distal UC $[10,11]$. Moreover, $n$-butyrate may exert anti-inflammatory effects by suppressing the activation of the TNF- $\alpha$-induced nuclear factor (NF)- $\kappa \mathrm{B}$ [33]. Taking the above observations together, we suggest that the anti-inflammatory effect of PG on DSS-induced colitis may be due to an increased concentration of $n$-butyrate in the colon.

The difference in the effects of PG and CS on colonic SCFA may be explained by the core protein of PG, which is composed mainly of serine and glycine $[1,2,13]$. The principal SCFAs that result from fermentation of both carbohydrates and amino acids are acetate, propionate, and butyrate, although formate, valerate, caproate, and the branched-chain fatty acids formed during the catabolism of branched-chain amino acids (valine, leucine, and isoleucine) are also produced in lesser amounts [34]. It has been reported that germinated barley foodstuff containing protein and hemicellulose significantly increases the concentrations of $n$-butyrate in normal rats and rats with DSS-induced colitis [35, 36].

Orally administered PG significantly increased the total concentration of SCFAs in colonic feces, whereas orally administered CS did not (Table 2). As shown in Fig. 6b, CS in the colon was completely degraded, suggesting that the degradation of CS started in the cecum, generating SCFAs in the cecum through the action of intestinal bacteria. CS is degraded by the anaerobic bacteria of the large intestine, mainly Bacteroides thetaiotaomicron [8, 9]. This bacterium also degrades pectin and other types of dietary fiber [15] and generates SCFAs [37]. Although the molecular size was slightly decreased, administered PG was detected in the rat colonic lumen (Fig. 6c), indicating that, compared with CS, $\mathrm{PG}$ is more resistant to degradation in vivo. Because of the attachment of CS chains to the protein backbone, the digestion of PG by B. thetaiotaomicron is slower than that of free CS in vitro [38]. Since SCFAs are absorbed readily by the intestinal mucosa [39], it is likely that SCFAs generated from CS by intestinal bacteria in the cecum are absorbed there and therefore unable to increase the concentration of SCFAs in the distal colon.

In conclusion, this is the first study to indicate that exogenously administered PG ameliorates experimental colitis, and we suggest that the anti-inflammatory effect of PG on DSS-induced colitis may be due to an increased concentration of $n$-butyrate in the colon, for which the structure of PG may be important. The present findings suggest that PG may have potential therapeutic applications for patients with colitis.

Open Access This article is distributed under the terms of the Creative Commons Attribution Noncommercial License which permits any noncommercial use, distribution, and reproduction in any medium, provided the original author(s) and source are credited.

\section{References}

1. Lena K, Ulf L (1991) Proteoglycans: structures and interactions. Annu Rev Biochem 60:443-75. doi:10.1146/annurev.bi.60.070191.002303

2. Renato VI, Alan DM (1996) Proteoglycans of the extracellular environment: clues from the gene and protein side offer novel 
perspectives in molecular diversity and function. FASEB J 10:598-614

3. Ade-Ajayi N, Spitz L, Kiely E, Drake D, Klein N (1996) Intestinal glycosaminoglycans in neonatal necrotizing enterocolitis. $\mathrm{Br}$ J Surg 83:415-418. doi:10.1002/bjs.1800830339

4. Murch SH, MacDonald TT, Walker-Smith JA, Levin M, Lionetti P, Klein NJ (1993) Disruption of sulphated glycosaminoglycans in intestinal inflammation. Lancet 341:711-714. doi:10.1016/ 0140-6736(93)90485-Y

5. Marossy K (1981) Interaction of the chymotrypsin- and elastaselike enzymes of the human granulocyte with glycosaminoglycans. Biochim Biophys Acta 659:351-361

6. Theoharides TC, Patra P, Boucher W, Letourneau R, Kempuraj D, Chiang G et al (2000) Chondroitin sulphate inhibits connective tissue mast cells. Br J Pharmacol 131:1039-1049. doi: 10.1038/sj.bjp.0703672

7. Morreale P, Manopulo R, Galati M, Boccanera L, Saponati G, Bocchi L (1996) Comparison of the antiinflammatory efficacy of chondroitin sulfate and diclofenac sodium in patients with knee osteoarthritis. J Rheumatol 23:1385-1391

8. Salyers AA (1979) Energy sources of major intestinal fermentative anaerobes. Am J Clin Nutr 32:158-163

9. Salyers AA, O'Brien M (1980) Cellular location of enzymes involved in chondroitin sulfate breakdown by Bacteroides thetaiotaomicron. J Bacteriol 143:772-780

10. Scheppach W, Sommer H, Kirchner T, Paganelli GM, Bartram P, Christl S et al (1992) Effect of butyrate enemas on the colonic mucosa in distal ulcerative colitis. Gastroenterology 103:51-56

11. Vernia P, Annese V, Bresci G, d'Albasio G, D'Inca R, Giaccari S et al (2003) Topical butyrate improves efficacy of 5-ASA in refractory distal ulcerative colitis: results of a multicentre trial. Eur J Clin Invest 33:244-248. doi:10.1046/j.1365-2362.2003. 01130.x

12. Okayasu I, Hatakeyama S, Yamada M, Ohkusa T, Inagaki Y, Nakaya R (1990) A novel method of reliable experimental acute and chronic ulcerative colitis in mice. Gastroenterology 98:694-702

13. Majima M, Takagaki K, Sudo S, Yoshihara S, Kudo Y, Yamagishi S (2001) Effect of proteoglycan on experimental colitis. In: Endo M, Harata S, Saito Y et al (eds) New developments in glycomedicine. Elsevier Science BV, Amsterdam, Netherlands, pp 221-224

14. Takagaki K, Kon A, Kawasaki H, Nakamura T, Tamura S, Endo M (1990) Isolation and characterization of Patnopecten mid-gut gland endo-beta-xylosidase active on peptidochondroitin sulfate. J Biol Chem 265:854-860

15. Cooper HS, Murthy SN, Shah RS, Sedergran DJ (1993) Clinicopathologic study of dextran sulfate sodium experimental murine colitis. Lab Invest 69:238-249

16. Bitter T, Muir HM (1962) A modified uronic acid carbazole reaction. Anal Biochem 4:330-334. doi:10.1016/0003-2697(62)90095-7

17. Miwa H, Hiyama C, Yamamoto M (1985) High-performance liquid chromatography of short- and long-chain fatty acids as 2-nitrophenylhydrazides. J Chromatogr 321:165-174. doi:10.1016/ S0021-9673(01)90433-9

18. Miwa H, Yamamoto M, Nishida T, Nunoi K, Kikuchi M (1987) High-performance liquid chromatographic analysis of serum long-chain fatty acids by direct derivatization method. J Chromatogr 416:237-245. doi:10.1016/0378-4347(87)80507-8

19. Gaudio E, Taddei G, Vetuschi A, Sferra R, Frieri G, Ricciardi G et al (1999) Dextran sulfate sodium (DSS) colitis in rats: clinical, structural, and ultrastructural aspects. Dig Dis Sci 44:1458-1475. doi:10.1023/A:1026620322859

20. Elson CO, Sartor RB, Tennyson GS, Riddel RH (1995) Experimental models of inflammatory bowel disease. Gastroenterology 109:1344-1367. doi:10.1016/0016-5085(95)90599-5
21. Ni J, Chen SF, Hollander D (1996) Effects of dextran sulphate sodium on intestinal epithelial cells and intestinal lymphocytes. Gut 39:234-241. doi:10.1136/gut.39.2.234

22. Tanaka M, Saito H, Kusumi T, Shimoyama T, Fukuda S, Morita T et al (2002) Biopsy pathology predicts patients with ulcerative colitis subsequently requiring surgery. Scand J Gastroenterol 37:200-205. doi:10.1080/003655202753416885

23. Stevceva L, Pavli P, Husband A, Matthaei KI, Young IG, Doe WF (2000) Eosinophilia is attenuated in experimental colitis induced in IL-5 deficient mice. Genes Immun 1:213-218. doi: $10.1038 /$ sj.gene.6363654

24. Hubscher T (1975) Role of the eosinophil in the allergic reactions II. Release of prostaglandins from human eosinophilic leukocytes. J Immunol 114:1389-1393

25. Zeiger RS, Twarog FJ, Colten HR (1976) Histaminase release from human granulocytes. J Exp Med 144:1049-1061. doi: 10.1084/jem.144.4.1049

26. Turk J, Maas RL, Brash AR II, Roberts LJ, Oates JA (1982) Arachidonic acid 15-lipoxygenase products from human eosinophils. J Biol Chem 257:7068-7076

27. Weller PF, Goetzl EJ (1980) The human eosinophil: roles in host defense and tissue injury. Am J Pathol 100:791-820

28. Beeken WL, Northwood I, Beliveau C, Baigent G, Gump D (1987) Eosinophils of human colonic mucosa: $\mathrm{C} 3 \mathrm{~b}$ and $\mathrm{Fc}$ gamma receptor expression and phagocytic capabilities. Clin Immunol Immunopathol 43:289-300. doi:10.1016/0090-1229(87)90138-3

29. Beeken W, Northwood I, Beliveau C, Gump D (1987) Phagocytes in cell suspensions of human colon mucosa. Gut 28:976-980. doi: 10.1136/gut.28.8.976

30. Cummings JH, Pomare EW, Branch WJ, Naylor CP, Macfarlane GT (1987) Short chain fatty acids in human large intestine, portal, hepatic and venous blood. Gut 28:1221-1227. doi:10.1136/ gut.28.10.1221

31. Vernia P, Gnaedinger A, Hauck W, Breuer RI (1988) Organic anions and the diarrhea of inflammatory bowel disease. Dig Dis Sci 33:1353-1358. doi:10.1007/BF01536987

32. Araki Y, Andoh A, Tsujikawa T, Fujiyama Y, Bamba T (2001) Alterations in intestinal microflora, faecal bile acids and short chain fatty acids in dextran sulphate sodium-induced experimental acute colitis in rats. Eur J Gastroenterol Hepatol 13:107112. doi:10.1097/00042737-200102000-00004

33. Venkatraman A, Ramakrishna BS, Shaji RV, Kumar NS, Pulimood A, Patra S (2003) Amelioration of dextran sulfate colitis by butyrate: role of heat shock protein 70 and NF-kappaB. Am J Physiol Gastrointest Liver Physiol 285:G177-G184

34. Macfarlane S, Macfarlane GT (2003) Regulation of short-chain fatty acid production. Proc Nutr Soc 62:67-72. doi:10.1079/ PNS2002207

35. Kanauchi O, Agata K, Fushiki T (1997) Mechanism for the increased defecation and jejunum mucosal protein content in rats by feeding germinated barley foodstuff. Biosci Biotechnol Biochem 61:443-448

36. Araki Y, Andoh A, Koyama S, Fujiyama Y, Kanauchi O, Bamba T (2000) Effects of germinated barley foodstuff on microflora and short chain fatty acid production in dextran sulfate sodium-induced colitis in rats. Biosci Biotechnol Biochem 64:1794-1800. doi: 10.1271/bbb.64.1794

37. Dongowski G, Lorenz A, Anger H (2000) Degradation of pectins with different degrees of esterification by Bacteroides thetaiotaomicron isolated from human gut flora. Appl Environ Microbiol 66:1321-1327. doi:10.1128/AEM.66.4.1321-1327.2000

38. Kuritza AP, Salyers AA (1983) Digestion of proteoglycan by Bacteroides thetaiotaomicron. J Bacteriol 153:1180-1186

39. McNeil NI, Cummings JH, James WP (1978) Short chain fatty acid absorption by the human large intestine. Gut 19:819-822. doi:10.1136/gut.19.9.819 